\title{
Superluminal Motion in the Gamma-Ray Blazar 3C 279
}

S. C. Unwin

Jet Propulsion Laboratory, Caltech, Pasadena, CA, U.S.A.

A. E. Wehrle \& W. Xu

IPAC, Jet Propulsion Laboratory, Caltech, Pasadena, CA, U.S.A.

A. C. Zook

Pomona College, Claremont, CA, U.S.A.

A. P. Marscher

Boston University, Boston, MA, U.S.A.

\begin{abstract}
The blazar $3 \mathrm{C} 279$ is one of the strongest extragalactic sources of $\gamma$-rays, and is also one of the best studied superluminal radio sources. Definitive testing of models of the broad-band spectral energy distribution, especially in the X-ray and $\gamma$-ray regions requires knowledge of the evolution of the spectrum with time. Within the context of the relativistic jet model, multi-wavelength monitoring of the parsec-scale radio jet is also required. We present here the first steps toward such a test, using extensive VLBI monitoring over a 13 year interval at $22 \mathrm{GHz}$, and $\gamma$-ray observations between 1991 and 1996 .
\end{abstract}

\section{VLBI Images of the Jet in $3 \mathrm{C} 279$}

The blazar 3C $279(z=0.538)$ has been the focus of one of the most extensive campaigns of monitoring with VLBI, covering the frequency range $5-43 \mathrm{GHz}$. Routine monitoring at $43 \mathrm{GHz}$, made possible by the NRAO VLBA, began in 1995. The most extensive dataset is a series of 18 images at $22 \mathrm{GHz}$, spanning the period 1984.75-1997.04. Figure 1 shows this series, omitting four additional images from 1984-1988, for which the time-sampling of the jet evolution is poor. Previous VLBI monitoring results are given by Wehrle et al. (1996). Points to notice: (1) the outermost component, moving at $4.3 \mathrm{~h}^{-1} \mathrm{c}$ is extremely longlived, showing no sign of fading after 13 years. (2) This component has a flat spectrum, as it shows strongly on $43-\mathrm{GHz}$ images. (3) Even with dense timesampling, structure variations near the core are complicated, apparently with a shorter timescale than the evolution farther out.

During multiwavelength monitoring in Jan-Feb 1996, 3C 279 reached its highest recorded level in $\gamma$-rays (Wehrle et al. 1997). A new component may have emerged from the core near that epoch - this component is well separated in the final epoch image (1997.04), but further monitoring is required to confirm its emergence in early 1996. The 1991 flare (Hartman et al. 1996) may be associated with blob that emerged from the core in mid-1991. Thus the evidence for associations is tentative, and likely to remain so until more is known about the long-term behavior of the source in $\gamma$-rays.

Acknowledgments. This work was supported by the NASA Long Term Space Astrophysics program. The National Radio Astronomy Observatory is a facility of the National Science Foundation, operated under a cooperative agreement by Associated Universities, Inc. 


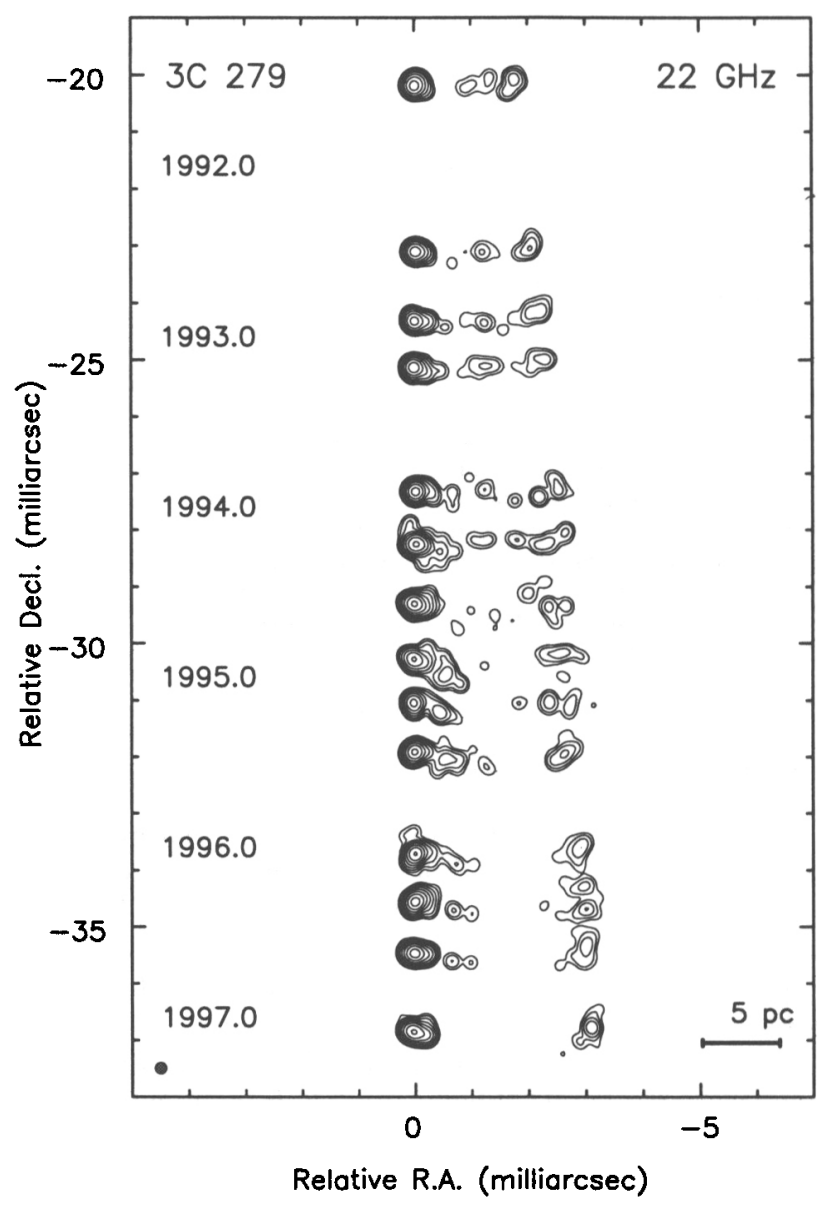

Figure 1. Multi-Epoch VLBI imaging of $3 \mathrm{C} 279$ at $22 \mathrm{GHz}$ (Wehrle et al. 1997). Vertical displacement is proportional to observing epoch (1 year $\equiv 3$ mas). Images were rotated $30^{\circ}$, to align the jet horizontally. Contours are in factors of 2 , starting at $75 \mathrm{mJy}$ /beam. Restoring beam is $0.2 \mathrm{mas}$ (dot at lower left); the resulting superresolution implies that north-south structure, perpendicular to the jet direction, is poorly constrained.

\section{References}

Hartman, R. C., et al. 1996. $A p J, 461,698-712$.

Wehrle, A. E., et al. 1996. in Blazar Continuum Variability, eds. H. R. Miller, J. R. Webb \& J. C. Noble, ASP Conference (San Francisco: Astron. Soc. of the Pacific), 430-435.

Wehrle, A. E., et al. 1997. $A p J$, submitted. 